\title{
Sound: An Effective Primary Medium of Communication for Individuals with Multiple Impairments
}

\author{
Sudharsan Iyengar ${ }^{1}$, Christine Bothun ${ }^{2}$ \\ ${ }^{1}$ Computer Science Department, Winona State University, $M N$ \\ ${ }^{2}$ Therapy Network, WI
}

\begin{abstract}
This paper describes the effective use of sound as a primary means of communication and teaching when someone is affected by multiple impairments. We present the results of the project that was conducted over 3 years to enable a student - referred to as $J$-with multiple impairments to learn and use basic life skills. J's impairments include visual, auditory, speech, motor skills, and possibly cognitive skills. Various strategies were used by OT and $J$ knew cause and effect but J could not communicate. We needed to teach discrimination so she can communicate on her own. Traditional teaching methods using visual cues and/or oral expressions were ineffective and hence we used sound - as an alternate to language - as a medium to teach J some basic life skills. We trained $J$ to associate sounds with objects. Subsequently through assessment processes we ascertained how well this skill was learned. Progressively we have used and assessed different sounds for different life skill activities - such as eating, wiping the face, drinking water, and such. $J$ now has the ability to request using programmed jelly bean switches.
\end{abstract}

\section{Introduction}

Traditional methods of teaching are ineffective when individuals have multiple impairments. According to the Individuals with Disabilities Education Act's (IDEA), multiple disabilities refers to "concomitant [simultaneous] impairments (such as intellectual disability-blindness, intellectual disability-orthopedic impairment, etc.), the combination of which causes such severe educational needs that they cannot be accommodated in a special education program solely for one of the impairments As stated in [1] "this group may require instructors to consider more elaborate accommodations". Students with visual impairment are taught and use Braille. They feel the Braille letters instead of looking at the printed word. Students hard of hearing learn and use sign language to communicate. But when they also have motor impairment they may not be able to use Braille or sign language effectively. So is the case when a student has visual and hearing impairments. Other techniques are required to supplement the issues posed by multiple impairments.

Students with severe cognitive and or speech language deficits but with vision use a picture system called PECS to communicate. Auditory therapy is used to achieve/seek attention and self-modulation in autistic and cognitively impaired subjects. There are many advantages to such therapy [2, 3, 4]. Unique and innovative techniques are required to supplement the issues posed by multiple impairments.

Simple sound or sound strings are devoid of linguistic complexities. Thus sound can be effectively used to teach elementary concepts and association. This method is shown to be effective as a Montessori Method to teach letter sounds [5]. The Montessori Method also uses basic shapes and colors to associate complex concepts as nouns, verbs, adjectives etc. when teaching language arts. The semantic concepts are associated with elementary and universally recognizable objects like sound or shapes.

Ability to identify and sort simple and regular objects is considered basic and used as a measure for pre-kindergarten screening.

Understanding this elementary human capability and engaging that to teach higher level concepts is one way we can overcome disabilities with multiple impairments [7], [8], [9]. For visually impaired it is the inability to view and consequently perceive and abstract the characteristics of the shapes. For the cognitively impaired the abstraction of the concept and the ability to associate linguistic expressions to objects, may be the factor.

Our project was to use elementary sound and sound strings to teach basic life skills to a student with multiple impairments and to study its effectiveness. There are many methods for teaching cause and effect, but not many to help students with multiple impairments learn to discriminate between two objects Augmentative and alternative 
communication is advocated and prescribed when working with such students [10]. A curriculum for early intervention for infants and young children with multiple impairments can be found in [11]. Furthermore a collaborative approach in these situations is warranted [12, 13, 14]. Every Move Counts [15] is "a sensory based approach to communication and assistive technology for individuals with significant sensory motor differences, developmental differences and autism" (www.everymovecounts.net). It was a program developed by Jane Korsten a speech and language therapist, Terry Foss, a special education teacher and Lisa Berry an occupational therapist. This program helps build on the interests and the abilities of students to help them communicate and build skills.

\section{Background}

We are working with a student - referred to as $\mathrm{J}$ with very limited vision capabilities, extremely limited limb and hand motor skills, extremely limited speech skill, and possibly cognitive deficits as well as hearing impairment, $\mathrm{J}$ does not communicate verbally or physically in any traditional way. $\mathrm{J}$ does not respond to verbal communication or physical gestures in the traditional way. Because of this combination of impairments - J struggled to learn. Her educational team used many strategies to reinforce cause and effect. She had switches to activate a blender to puree her lunch, activate a fan and play music. Despite her great progress with understanding cause and effect, she struggled with learning to differentiate between objects. When attempting to teach the ability to ask for a bite of food or drink, she would grab the object with one hand and bang it on the table. The occupational therapist tried yellow and red switches with the words, "bite" and "drink" with little progress. She did not reach for the object that was consistent with her behavior. After years of working with different approaches and not making significant progress with differentiating between objects, a new strategy needed to be tried. The occupational therapist knew that she was interested in sound. $\mathbf{J}$ cannot respond to verbal communication or physical gestures in the traditional way. Therefore, the occupational therapists assigned to assist her at school have not been able to communicate and/or receive feedback from her. This difficulty had been there since birth and she is now a teenager.

During one of the discussions between the authors - one a computer science professor involved in foundational artificial intelligence research and the other an occupational therapist grappling with ways to assist $\mathbf{J}$ - the idea to use alternate mode to train and teach $\mathbf{J}$ was conceived. The foundational research in AI by the first author deals with identifying cognitive primitives that form the foundational framework for higher order intelligence. One of the approaches is to identify cognitive primitives that are devoid of semantics and then superimpose domain specific semantics to use these primitives. Sound and consequently music is one such medium used in cognition but semantics is not necessary. The use of audible techniques have been used over thousands of years for teaching music as well as Vedic and Gregorian chants [16]. Analyzing languages and the phonemes used for elementary concepts yields fundamental characteristics that can be used to create new pseudo natural languages [16].

Thus the use of sound strings as an alternative mode of communication for $J$. This intervention technique is devoid of linguistic complexity and does not involve visual gestures. We first enable $\mathbf{J}$ to associate regular objects with regular sound strings and thus enable $\mathrm{J}$ to recognize and/or sort these objects. Subsequent to this we apply similar process to teach life skills.

Our project used a very simple custom software that, upon the therapist's selection plays sound strings for associated objects. $J$ was trained with the assistance of the OT. After the training we observed for voluntary reaction to the sound strings to assess J's learning.

\section{The Project}

The project was implemented to train and subsequently study the response, in stages as described below. Evidence of learning was monitored for the effectiveness.

We developed a prototype software (SAM) that plays selected musical strings. Associated in the UI with this selection is an object. This was used by the examiner to select an object and play the corresponding sound (that $\mathrm{J}$ can hear) during the training of hand-to-object by the OT or to observer J's reaction during assessment. The system has various selection options and enables the process to be repeated without noise. The software was modified when objects and their sounds are changed during the different stages.

SAM is implemented in Java using the JFugue sound package. Sound output can be controlled and presented to $\mathbf{J}$ via speakers or earphones. $\mathbf{J}$ was trained, in stages, by the examiner/OT. Subsequently J's response to playing a sound was observed for evidence of learning. During the assessment process upon a positive response by $\mathbf{J}$ a musical piece was played - as a "Good Job" reward. 
The following describes the different stages of the project.

\subsection{Teach that the sound has meaning}

Teaching Strategy: Hand under hand reach for object that matches sound sequence. The trainer assists with hand under J's hand to reach some object that matches a sound sequence.

Assessment: Behavior that is evidence of learning: Look for intention to reach or volitional movement when $\mathbf{J}$ hears a sound. Indicative that $\mathbf{J}$ has learned that sound sequence has meaning.

Prior to this stage $\mathrm{J}$ listened to music. $\mathrm{J}$ has been listening to music passively without realizing that the sound can have meaning. The intent of this stage was to create this understanding - that sound has a purpose.

\subsection{Teach specific sound is associated with an object}

Teaching strategy: Hand under hand reach for object that matches sound sequence. The trainer assists with hand under J's student hand to reach an object that matches a sound sequence.

Assessment: Behavior that is evidence of learning: Look for holding object that matches the sound played. This is indicative that $\mathrm{J}$ has learned that a specific sound sequence has specific matching object.

Success in this stage indicated that $\mathbf{J}$ was aware that sound has specific meaning. The intent of this stage is to create an understanding that a sound sequence matches a meaningful objects.

\subsection{Teach different sound sequence mean different objects}

Teaching strategy: Hand under hand reach an object that matches the sound sequence that is played. The trainer assists with hand under J's hand and reach the object that matches the sound sequence. We did this step with just two objects in the training process (as $\mathbf{J}$ has very limited reachability) placed in specific locations.

Assessment: Behavior that is evidence of learning: Look for $\mathrm{J}$ to reach and grasp the object that matches sound sequence played. The voluntary reach towards the appropriate object is observed. The objects are placed at the same location as during the training.
Indicative that $\mathbf{J}$ has learned that different sound sequences have different meaning and that they match different objects.

$70 \%$ or more time/frequency of correct response is use as a success of demonstrating this behavior.

By this stage $J$ is aware that a sound sequence has specific meaning. The intent of this stage was to create an understanding that different sound sequences mean different objects.

\subsection{Teach to act in a specific way to sound - sorting objects}

Teaching strategy: Hand under hand reach, grasp, carry and release of the object that matches the sound sequence from the table to a bucket. The trainer assists with hand under J's hand to reach matching object and drop in a bucket.

Assessment: Behavior that is evidence of learning: $\mathrm{J}$ drops the correct object into the bucket. Indicative that $\mathbf{J}$ was able to match objects to matching sound sequences, and was able to use her limited motor skills to drop the objects into a bucket.

Given that $\mathbf{J}$ has this motor skill, we know if the third stage is successful then $\mathbf{J}$ should be able to pass this stage. If $\mathbf{J}$ doesn't pass this - it is evidence that the inability is not due to the motor skill, but due to the inability to match the sound sequence to the proper object. Also, we expand the cognitive ability to act on the sound.

\subsection{Teach sound sequence to sort recyclable objects}

Teaching strategy: Training similar to Fourth stage, except the objects used are a bottle and a can. Sorting this using the "Beee" and "Caaa" sound is training to teach recycling of the objects. These are two new sounds and objects.

Assessment: Behavior that is evidence of learning: $\mathbf{J}$ will drop the correct object into the bucket in response to the corresponding sound. Indicative that $\mathbf{J}$ is able to match objects to matching sound sequences, and is able to use motor skills to sort recyclable objects.

Given that $\mathbf{J}$ can match sound to object from Fourth stage, we use this stage to teach vocational skill of sorting recycling objects. If J doesn't pass this - it is evident that there exists an inability to distinguish different sounds for different objects. Again $70 \%$ accuracy is used to demonstrate learning. 
After successful completion of these stages the next steps were to teach life skills - viz. to communicate for water, food, or to be moved.

\section{Sound, Shape and their Co-relation}

This project involved simple co-relation between sound strings and objects. The sound strings map to a conceptual object. To begin with the number of notes (equal duration) played equals the number of sides of a shape.

A square object had a sound sequence of four (4) notes. A sphere was associated with a single smooth note that starts at the note $\mathrm{C}$ goes all the way to note $\mathrm{C}$ at the next octave and returns back to the starting $\mathrm{C}$ note.

Subsequently different note strings are used for different objects. We used two objects at a time for training and assessment.

In order to accommodate the auditory capabilities and affinities of subjects we are using a variety of instruments with a high and/or a low pitch notes to make it comfortable. Sounds from instruments like the Violin and Cello, Flute, and Piano were used for the project.

\section{The Software (SAM)}

The software (SAM) is menu driven and plays selected musical strings. It can be customized to include images of various objects and play different recorded sounds. Associated in the UI is the selection of an object. This is used by the examiner to play the sound.

The OT uses this software to select an object, play the corresponding sound and train using handto-object or observe J's reaction during assessment. The voluntary reaction to the sound strings is observed to assess J's learning. The software has various selection options and enables the process to be repeated.

SAM is implemented in Java using the JFugue sound package. Sound output can be controlled and presented to $\mathbf{J}$ via speakers or earphones. $\mathbf{J}$ was trained, in stages, by the examiner/OT. During the assessment process upon a positive response by $\mathrm{J}$ a musical piece was played - as a "Good Job" reward.

Figure 1 shows a sample screen shot of SAM.

There are additional settings for volume, and enabling and playing a sequence of strings with pause or other markers in between. Thus it allows us to use customize the notes, duration, and instruments used for the sound sequences.

\section{Implementation and Data Collection}

The study process was implemented as follows:

- Stage I was used for training and assessment as described. We used two or more sessions for this stage.

- There are multiple sessions of Stage II. Each session had three steps. Step 1 was base-line assessment. Step 2 was training. And Step 3 was for assessment. Results from step 1 helped us measure if there was retention between sessions. Results of step 3 enabled us to understand/measure if training helped in the teaching process.

- $\quad$ Stage III is training and assessment as described. Multiple sessions for this stage were conducted as in Second Stage.

- There were multiple sessions of Stage IV. Again, each session had three steps. Step 1 for base-line assessment. Step 2 for training. And Step 3 for assessment. Again results from step 1 helped us measure if there was retention between sessions. Results of step 3 helped us understand/measure if training has helped in the teaching process.

- Training and assessment of the stage IV was used to validate ability to sort.

- Training and assessment of the Stage V stage was used to validate ability to distinguish different sounds and sort recyclable objects.

Data during the sessions was collected on a data form shown in Figure 2. This data was subsequently used for further analysis. Forms include the Subject ID, Session Description (Assessment or Training), Session Number, Date, Start and End Time, Shapes used.

Additionally, these forms include Measured Data. These are anticipated result such as the object were a) held or not, b) sorted or not. These measurements were recorded on the form at least five times per session.

There is also space for the examiner to include any additional appropriate comments. These include comments on the suitability of the examining environment, subject response and progress as perceived by the examiner. Figure 2 shows the data form used to record J's performance during training and assessment.

For each stage described above, the training/teaching was done to the satisfaction of the trainer/examiner. Due consideration was given to the duration between training and assessment. 
When the assessments indicate high level of learning and if repetition was deemed redundant, then

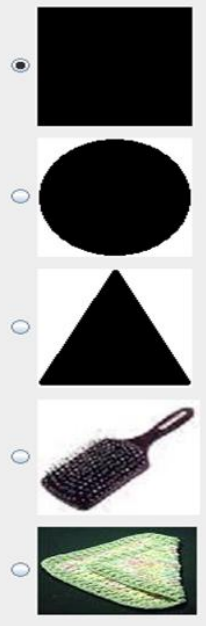

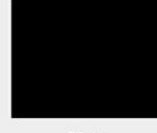

Flute

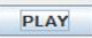

GOOD JOB

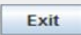

Tuba

Piano

- Flute

Cello

Figure 1. SAM - Sample User Interface

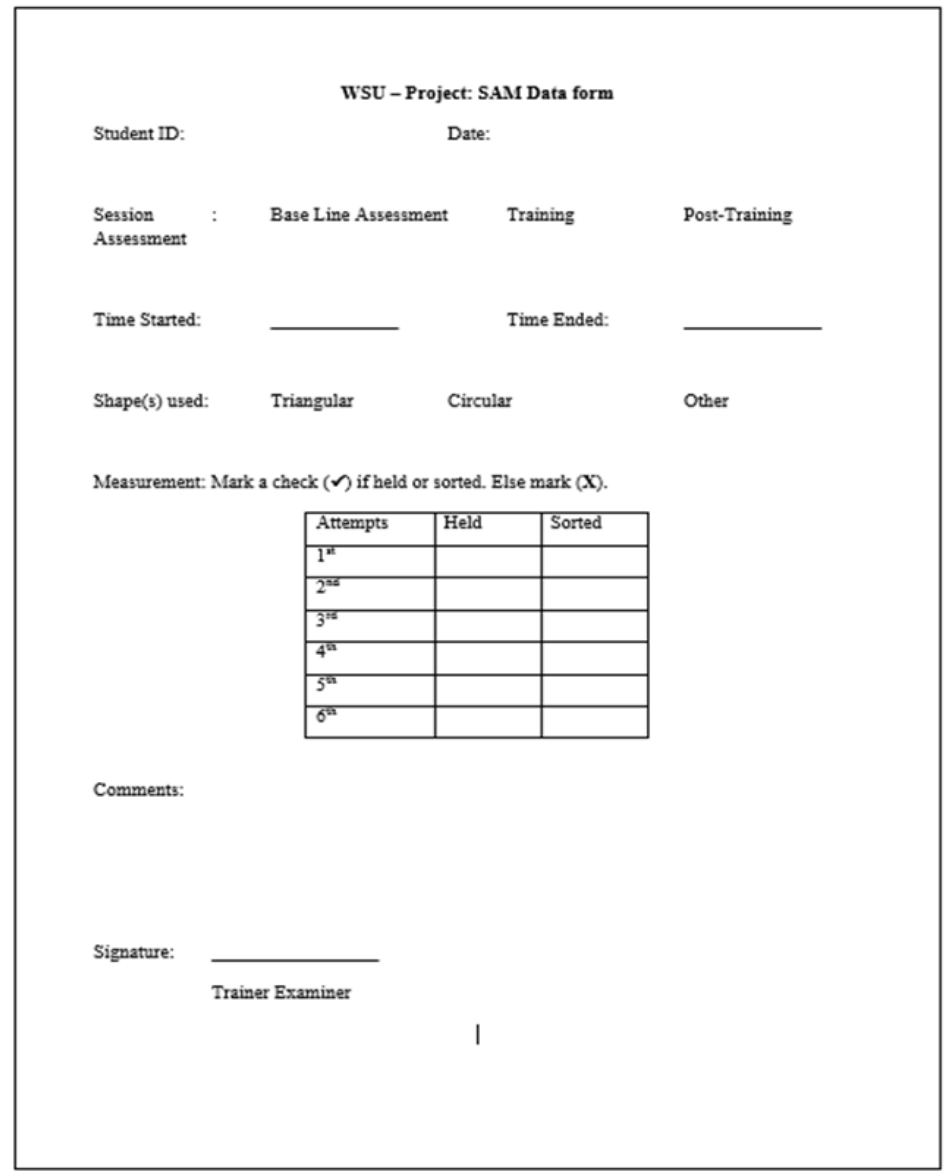

Figure 2: Data Form used with SAM 
that information was noted on the form, and the study proceeded to the next stage. When the assessments indicated low level of leaning then the study for that stage was repeated.

The results of the field tests were used to understand the effectiveness of sound as a medium for communication in assisting and teaching visually impaired students with cognitive and/or speech and language impairments.

Subsequent study has helped us teach $\mathrm{J}$ to reach for brush to comb hair, reach for towel to wipe face, and with the use of button request water or food by pressing the appropriate programmed jelly bean switches.

\section{Evaluation}

We evaluated the training and assessment sessions to make changes that helped the process conducive and adaptive to the J's needs. Some of the factors that affected the sessions, and the results thereof are: J's ability to participate - due to health or other reasons, mode of presentation of the sound strings, objects and their design, duration of the time, and frequency, of the tests. Therefore, many of the training and assessment sessions were video-taped for the sole purpose of observation, re-examination, and for proper collection of training and assessment data. At the end of each sessions the results of the sessions were qualitatively summarized and retained by the examiner. Based on the result the sessions were either repeated or the progress noted and preparations for the next session/stage is initiated.

\section{Conclusion}

The first thing that SAM taught $\mathbf{J}$ was to listen. With headphones on, a sound occurred with the presentation of an object. This way $\mathrm{J}$ learned that a sound symbolized an object and that sound had meaning. From this stage $\mathrm{J}$ progressed towards listening to sounds to discriminate between two objects. Due to J's physical limitations, consistent accurate physical reach is difficult. At the end of this stage, J. could sort circle (ball) and square (cube) objects with up to $75 \%$ accuracy. J. is working on sorting activities where different objects go in different places. This task is more difficult due to the complexity of the motor movements to carry and release objects. When presented with a can and bottle, $\mathrm{J}$ can put them into separate containers providing her a vocational skill of sorting recyclables. J's accuracy fluctuates between $60-80 \%$.
In addition to the specific skills related to the trials, J. made additional gains. Now, J enjoys listening. $\mathrm{J}$ can listen and pay attention up to thirty minutes on a story. It is clear that $\mathrm{J}$ is listening better as $\mathbf{J}$ expresses discontent with the person reading the story stops. $\mathbf{J}$ has started using a communication device (programmable jelly bean switch) where $\mathrm{J}$ grabs an object to slide across the device to say a word. J uses this device to make choices for a drink or bite of food during snack time. Initially, $\mathbf{J}$ was dependent with eating with a staff person bringing the food to J's mouth and placing it there. Now, J can bring a spoon to her mouth and pulls against her teeth to clear it. The speech and language therapist is working on mouth closure. J's hand-washing routine has expanded to include the paper-towel dispenser. $\mathrm{J}$ can independently activate the paper-towel dispenser. With the sound it makes when it dispenses, J. knows to reach for the paper towel, tear the paper towel and throw it away. J continues to need support to dry hands. J consistently turns off the light when leaving an area, although may need multiple attempts due to the motor aspect of the task. $\mathbf{J}$ is beginning to work on functional mobility. Although $\mathrm{J}$ is confined to a wheelchair and unable to operate it due to bilateral motor challenges and cortical visual impairment, $\mathbf{J}$ is beginning to reach for an object that symbolizes movement. So on a walk, J can let the person pushing know that $\mathrm{J}$ wants to go forward.

Subsequent study has helped us teach $\mathrm{J}$ to reach for brush to comb hair, reach for towel to wipe face, and with the use of jelly bean switch to request water or food by pressing the appropriate switch.

There are multiple communication devices. What is unique about our study is the ability to teach discrimination which is a step beyond cause and effect that is involved with many communication devices that require individuals to ask or request an object. Our approach resulted in the instructional benefit that $\mathrm{J}$ was able to voluntarily communicate after learning discrimination.

\section{References}

[1] Johnson, David, "Teaching Students with Disabilities", David Johnson John Brown University, Essays from Excellence in Teaching, 2002, pp. 16-19.

[2] Gewanter, Sarah, 'What is Auditory Integration Training?', http://www.vision3d.com/adhd/\#AIT. Access Date: 28 July, 2015.

[3] Roux, Maude Le, http://www.atotalapproach.com/, “A Total Approach - Equipping the Child", Access Date: 28 July, 2015. 
[4] Frick Sheila, "What is Therapeutic Listening?", http://www.vitallinks.net/, Access Date: 28 July, 2015.

[5] Melissa, "The Montessori Method for Teaching the Letter Sounds", http://vibrantwanderings.com/2012/08/themontessori-method-for-teaching-the-letter-sounds.html,

Publish Date: August 27, 2012; Access Date: July 28, 2015.

[6] Hosken, Cheryl K, "Severe and Multiple Disabilities", http://www.agape-biblia.org/plugins/pract-

ministries/Lect106.htm, Publish Date: 2008, Access Date: 28 July, 2015.

[7] Watson, Sue, "Multiple Disabilities", http://specialed.about.com/od/multipledisabilities/a/multipl e.htm, Copyright 2015, Access Date: 28 July, 2015.

[8] Texas Council of Developmental Disabilities, "Multiple Disabilities", http://www.projectidealonline.org/v/multipledisabilities/, Copyright 2013, Access Date: 28 July, 2015.

[9] Annapolis Valley Regional School Board, "Meeting the Needs of Students with Severe and/or Multiple Disabilities":, $\quad$ http://www.nsnet.org/start/severe.pdf, Copyright 2009, Access Date: 28 July, 2015.

[10] Downing, J.E. (2002), "Including students with severe and multiple disabilities in typical classrooms: Practical strategies for teachers" (2nd ed.). Baltimore, MD: Paul H. Brookes. (Telephone: 800.638.3775. Web: www.brookespublishing.com).

[11] Klein, M.D., Chen, D., \& Haney, M. (2002). "PLAI (Promoting learning through active interaction): A guide to early communication with young children who have multiple disabilities", Baltimore, Paul H. Brookes. (See contact information above.).

[12] Orelove, F., \& Sobsey, D. (1996), "Educating children with multiple disabilities: A transdisciplinary approach (3rd ed.). Baltimore, MD: Paul H. Brookes.”, (See contact information above.).

[13]Rainforth, B., York, J., \& Macdonald, C. (1997). Collaborative teams for students with severe disabilities: Integrating therapy and educational services (2nd ed.). Baltimore, MD: Paul H. Brookes. (See contact information above.)

[14] Sasaki, et. Al., The Development of Music Presentation System by Two Vibrators, ICCHP 2014, pp. 602-605.
[15] Jane Korsten, Terry Foss,Lisa Berry, "Every Move Counts, Clicks and Chats", http://www.everymovecounts.net", Access Date 10/3/2015.

[16] Sudharsan Iyengar, (2006), "A Framework for Generating Chants Using Mnemonic Capabilities", Proceedings of Sound and Music Computing, Marseilles, pp 128-130.

[17] Sudharsan Iyengar, (2008), "Formulating PseudoNatural Language", Proceedings of ITNG-NLP, Las Vegas, pp 662-667. 